\title{
Numerical Study of Nucleate Boiling Flows Using ANSYS Fluent
}

\author{
Mohammed Azhar \\ ANSYS UK Ltd. \\ 6 Europa View, Sheffield S9 1XH, UK \\ mohammed.azhar@ansys.com
}

\begin{abstract}
In the recent decade, computational fluid dynamics has emerged as a tool to improve our understanding of boiling heat transfer. In the nuclear industry, for ins instance, high fidelity modelling of the critical heat flux has stimulated much interest in modelling boiling flows. In this paper, we evaluate the accuracy of the boiling models implemented in the ANSYS Fluent software against experimental data. The boiling model is based on the heat flux partitioning approach and accommodates the heat flux due to single-phase convection, quenching and evaporation. Extensive validations carried out for a range of $2 \mathrm{~d}$, and $3 \mathrm{~d}$ boiling flows demonstrate that the boiling models can correctly predict the vapour volume fraction distribution. The numerical results in all the cases are in good agreement with the experimental data.
\end{abstract}

Keywords: ANSYS Fluent, boiling heat transfer, computational fluid dynamics, two-phase flow.

\section{Introduction}

Boiling occurs in many industrial applications: nuclear reactors, heat exchangers, steam generators. Prediction of the vapour volume fraction profiles and other design parameters in these boiling flows is essential for the design and safety analysis of such systems. In the nuclear industry, for instance, interest in the prediction of two-phase flow behaviours is of great importance to the safety analysis of nuclear reactors.

The prediction of vapour volume fraction in boiling flow in vertical pipes and channels has been the subject of numerous studies in the literature. Most of these studies are based on empirical correlations. The major drawback of these correlations is that they are valid only for the specific conditions in which they are tested. The Computational Fluid Dynamics (CFD) software, on the other hand, allows the detailed analysis of the two-phase flow and heat transfer. In this paper, we present results of numerical analyses aimed at validating the Rensselaer Polytechnic Institute (RPI) [1] boiling model in ANSYS Fluent [5].

\section{Technical background}

The Eulerian multiphase model in ANSYS FLUENT solves separate continuity and momentum equations for each of the phases. The energy equation is solved only for the liquid phase, and the vapour phase is assumed to be at saturation temperature. The energy and momentum interfacial coupling terms are modelled using correlations listed in Table 1. Turbulence is modelled for each phase separately using the SST $k-\omega$ model. The boiling is modelled, which a subset of the Eulerian multiphase model, is based on RPI wall heat flux partitioning model. The heat flux from the wall is resolved into three components such as the convective, quenching and evaporative heat fluxes. Again, these components are calculated from flow and temperature fields along with other correlations for nucleation site density, departure frequency, and departure diameter shown in Table 1.

\section{Model Testing and validation}

To evaluate the RPI Boiling model in ANSYS Fluent, we consider an upward flow of subcooled water through simple tubular systems. The first example is an upward flow of subcooled liquid through a heated vertical pipe, experimentally studied by Bartolemei et al. [2][3]. The second example is a turbulent subcooled boiling flow of water through a vertical square sectioned duct, which was experimentally studied by Pierre et al. [4]. 
Table 1: Model for interfacial exchange terms used in the present simulation.

\begin{tabular}{|c|c|}
\hline Physics & Model \\
\hline Drag force & Ishii \\
\hline Lift force & Moraga \\
\hline Wall lubrication & Hoskowa \\
\hline Turbulent dispersion & Burns \\
\hline Turbulent interaction & Sato \\
\hline Interfacial area & Particle \\
\hline Nucleation site density & Lemmert \& Chawla \\
\hline Bubble diameter at departure & Tolubinski \& Kostanchuk \\
\hline Bubble departure frequency & Cole \\
\hline
\end{tabular}

\subsection{Example}

\subsubsection{Heated vertical pipe}

We first validate the simulation model against the classic experiments of Bartolemei et al. The experimental set-up is illustrated schematically in Figure 1. The subcooled water enters the pipe at the bottom at pressure $P_{\text {in }}=6.89 \mathrm{MPa}$ and subsequently boils due to the constant heat flux supplied from the pipe walls. In the heated lower section of the pipe subcooled boiling occurs and steam is generated. The section above is adiabatic, and vapour condensation occurs due to the mixing of the vapour with the still subcooled liquid core.

The numerical simulation exactly mirrors the experiment in Bartolemei et al. With flow being axisymmetric, only half of the pipe is considered. The mesh resolution is approximately 25000 cells; this is in line with ANSYS best practice guidelines for modelling boiling flows. The flow behaviour and heat transfer in the system is modelled as a two-phase mixture of liquid and gas. The flow is assumed to be steady state. The liquid phase properties can vary with the fluid temperature, while the vapour phase assumes saturation properties. The heat flux is applied to the heated wall surface, and the wall thickness is not resolved as depicted in Figure 1. The coupled multiphase solver with pseudo transient treatment and quick discretisation for spatial discretisation are used in all simulations, and the numerical predictions are validated against two tests. The controlling parameters for these tests are listed in Table 2.
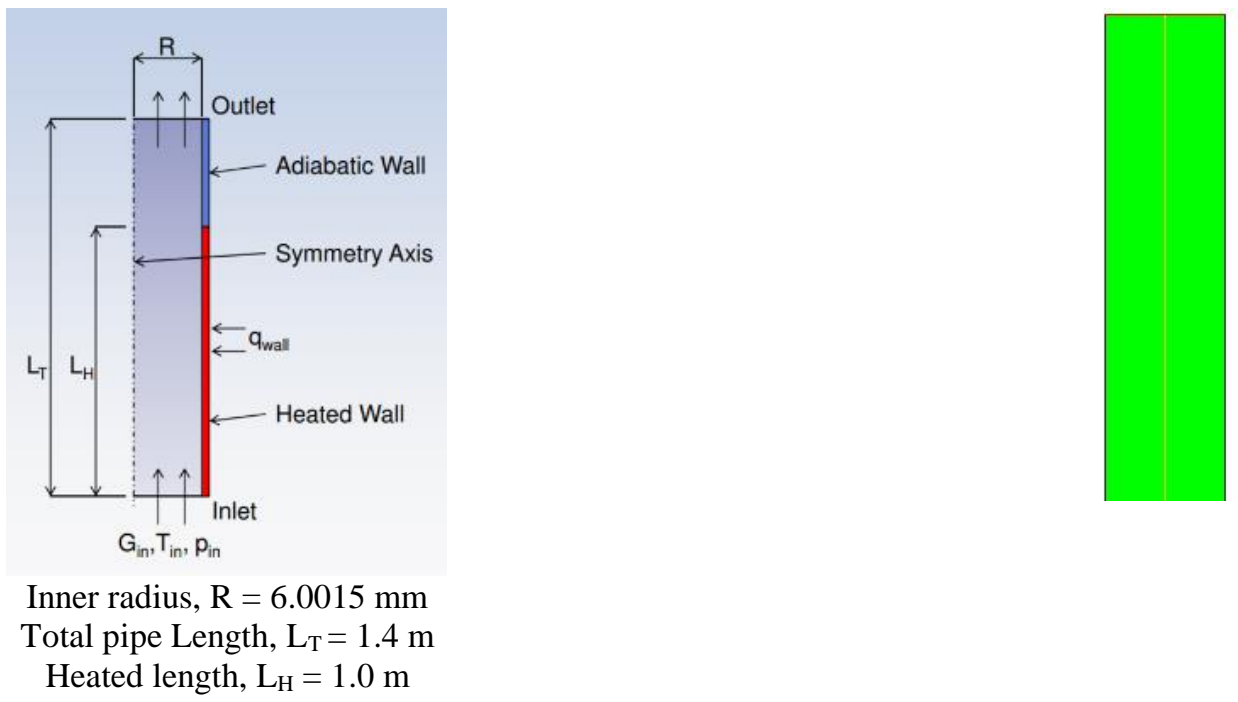

Fig. 1: Schematic of the experimental layout and the computational mesh considered for Example 1. 
Table 2: Flow parameters for Bartolemei et al. experiments.

\begin{tabular}{|c|c|c|c|c|}
\hline Experiment & $\boldsymbol{q}, \mathbf{M W} / \mathbf{m}^{\mathbf{2}}$ & $\boldsymbol{G}, \mathbf{~ k g} /\left(\mathbf{m}^{\mathbf{2}} \cdot \mathbf{s}\right)$ & $\boldsymbol{T}_{\boldsymbol{l}}, \mathbf{K}$ & $\boldsymbol{\Delta T}_{\text {sub }}, \mathbf{K}$ \\
\hline 2 & 1.2 & 1500 & 495 & 63 \\
\hline 5 & 0.8 & 1000 & 503 & 55 \\
\hline
\end{tabular}

Figure 2 shows the predicted axial temperature profiles at three radial locations. As can be seen, the liquid temperature increases in the radial direction due to wall heating. On reaching the saturation conditions, the temperature profiles suffer a reduction in the slope and tend towards a plateau.

Figure 3 shows the radial temperature profiles at three axial locations. It is evident that there are significant temperature differences between the centre and wall region at $\mathrm{x}=0.5 \mathrm{~m}$. Temperature is high close to the wall where the boiling is taking place and is below the saturation value at the centre of the pipe. This difference decreases along the pipe length, and a more uniform temperature distribution is seen at $\mathrm{x}=1.3 \mathrm{~m}$. Figure 4 shows the radial distribution of axial fluid velocity at same axial locations. The increase in the fluid speed is attributed to the rise in the vapour volume fraction along the pipe length.

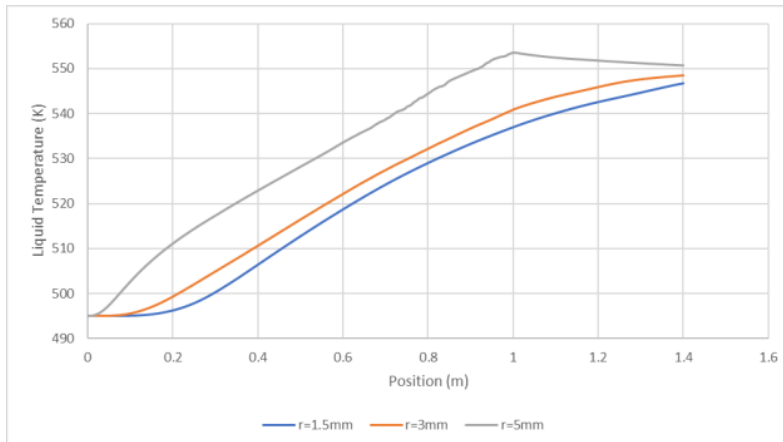

Experiment 2

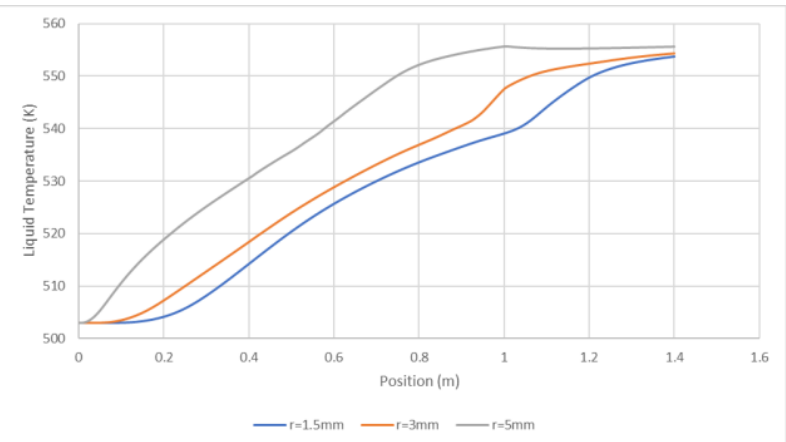

Experiment 5

Fig. 2: Computed axial temperature profiles at three radial locations.

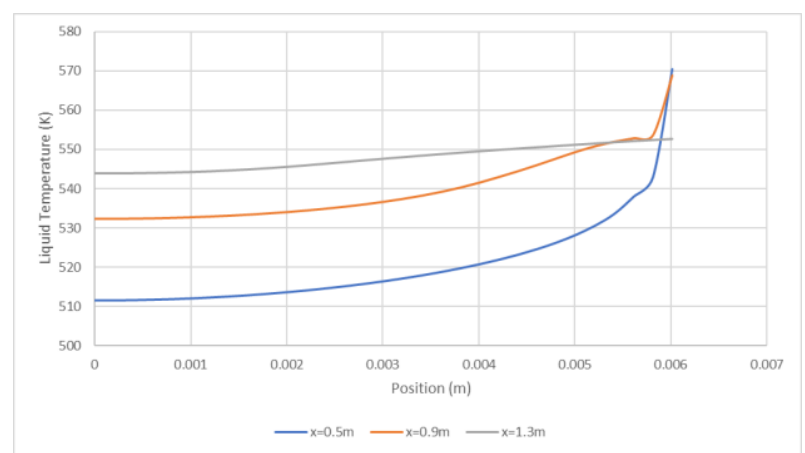

Experiment 2

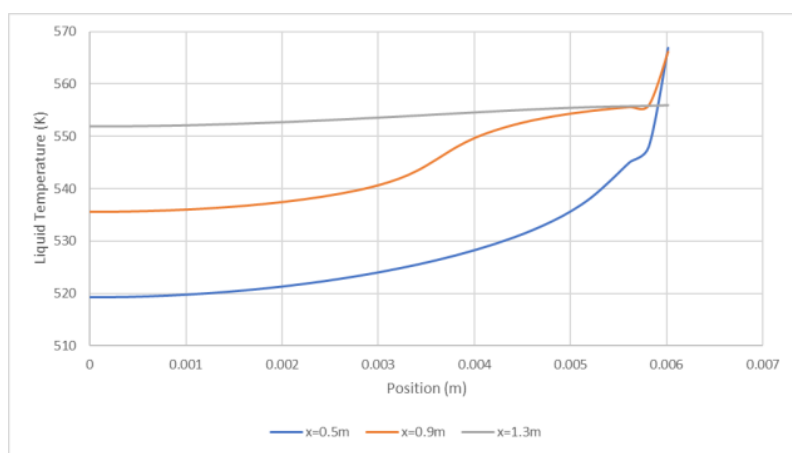

Experiment 5

Fig. 3: Computed radial temperature profiles at three axial locations. 


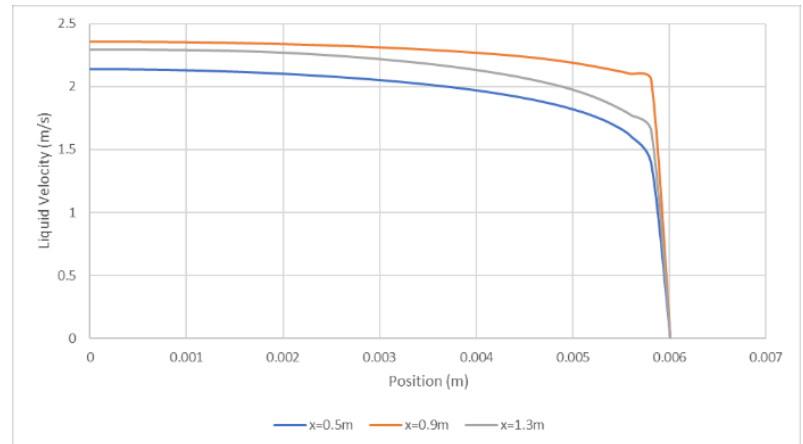

Experiment 2

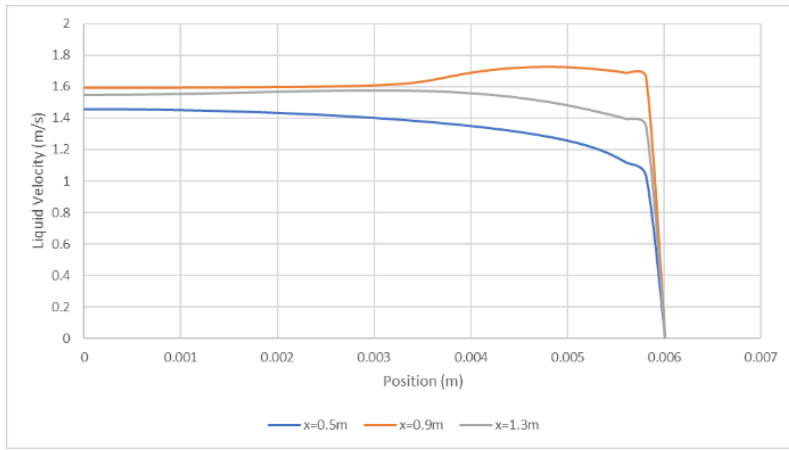

Experiment 5

Fig. 4: Estimated radial velocity profiles at three axial locations.

Figure 5 compares the predicted area averaged axial vapour volume fraction distribution with the experimental data of Bartolemei et al. The experimental and numerical volume fraction are in reasonably good agreement, although the discrepancy in some sections is seen. A closer agreement is obtained for the numerical simulation of experiment 2 , especially in the lower section. Simulations of experiment 5 , on the other hand, show a poor prediction of the volume vapour fraction. This suggests that perhaps vaporisation and condensation models need to be improved to handle a broader range of flow parameters.

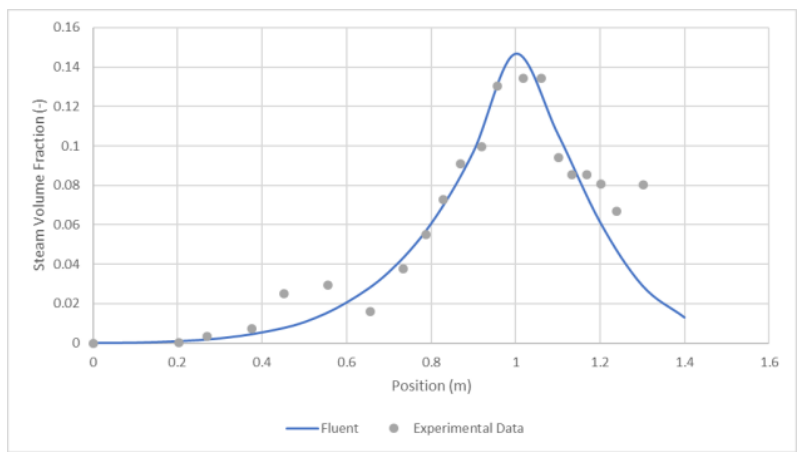

Experiment 2

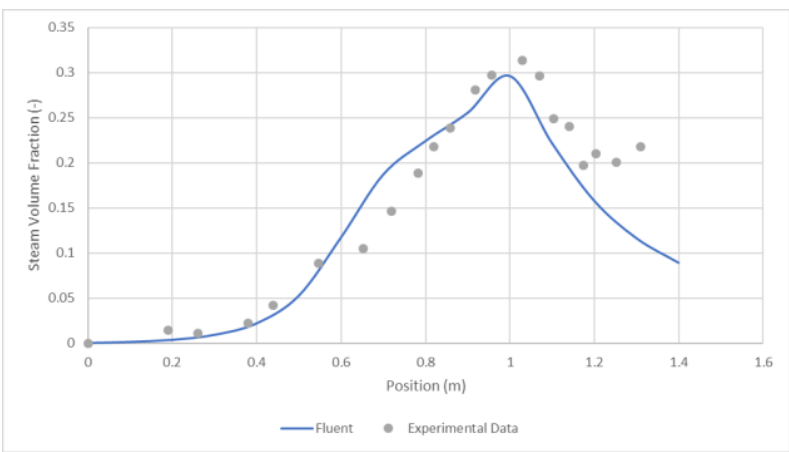

Experiment 5

Fig. 5: Average axial vapour volume fraction profiles.

\subsection{Boiling Flow in a Rectangular Sectioned Duct}

We now compare the numerical simulation for boiling vertical flow rectangular channel with experimental data of Pierre et al. We consider a vertical duct of $1.5494 \mathrm{~m}$ length, $44.4 \mathrm{~mm}$ width and $11.0 \mathrm{~mm}$ depth. The subcooled water enters the duct at the bottom and subsequently boils due to the constant heat flux supplied from the walls in heated section $0.2921 \mathrm{~m}$ from the inlet. The heated section extends $1.2273 \mathrm{~m}$ in length. Pierre et al. carried-out experiments under ten different operating conditions; however, we only are considering four simulations, and the flow parameters for these are listed in table 3. 
Table 3: Experimental Condition.

\begin{tabular}{ccccc}
\hline Experiment & $\begin{array}{c}\text { Pressure } \\
(\mathbf{p s i a})\end{array}$ & $\begin{array}{c}\text { Inlet Velocity } \\
(\mathbf{f t} / \mathbf{s})\end{array}$ & $\begin{array}{c}\text { Heat Flux } \\
\left(\boldsymbol{B t u}_{\mathbf{h}} \mathbf{~ f t} \mathbf{~}^{\mathbf{}}\right)\end{array}$ & $\begin{array}{c}\text { Inlet Subcooling } \\
\left({ }^{\boldsymbol{}} \mathbf{F}\right)\end{array}$ \\
\hline $\mathbf{1}$ & 200 & 3.78 & 2.28 & 0.5 \\
\hline $\mathbf{3}$ & 400 & 2.52 & 4.56 & 4.8 \\
\hline $\mathbf{5}$ & 400 & 3.78 & 6.84 & 1.2 \\
\hline $\mathbf{7}$ & 600 & 3.78 & 9.12 & 4.2
\end{tabular}

Figure 6 shows the contours of the vapour volume fraction at several cross sections along the duct for experiment 7 . It is evident that the subcooled water starts evaporating immediately after entering the heated section. The vapour production is high near the heated surface where boiling is taking place. The vapour volume fraction increases along the duct length and the exit, it peaks with the maximum value of 0.57 .
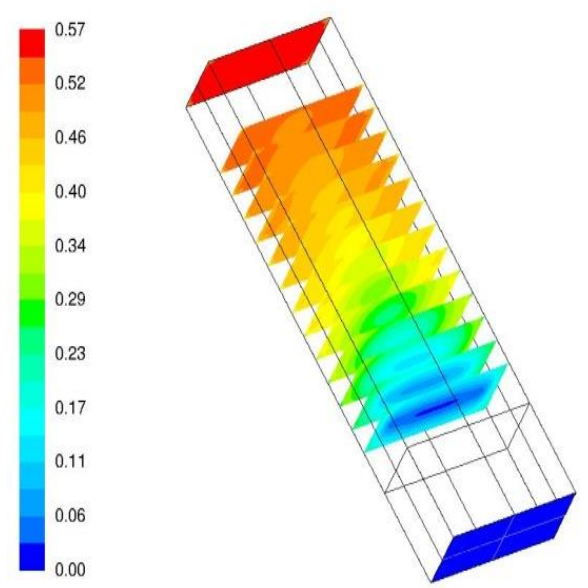

Fig. 6: Contours vapour volume fractions distributions for Experiment 7.

Figure 7 compares the predicted and experimental area averaged vapour volume fraction profiles along the duct. While these simulations do a reasonable job at predicting the onset of nucleate boiling and vapour volume fraction trends, they tend to overpredict the vapour volume fraction. This suggests that perhaps vaporisation and condensation models should be improved to handle a broader range of flow parameters. Overall a reasonable agreement between numerical and experimental data was obtained in calculations for boiling and condensation processes. 


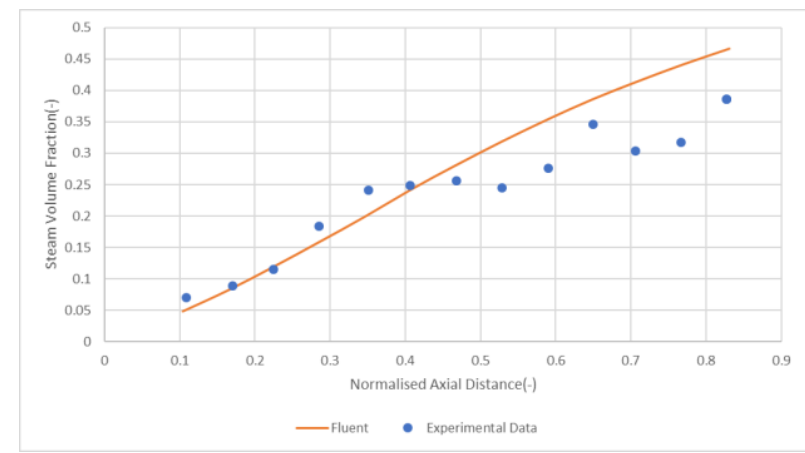

Experiment 1

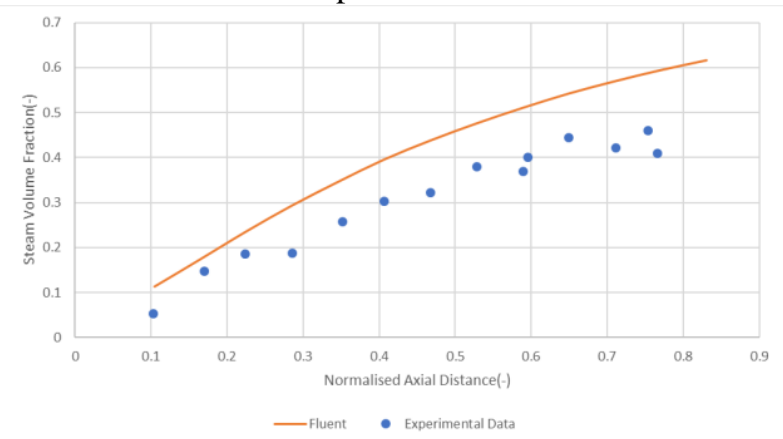

Experiment 5

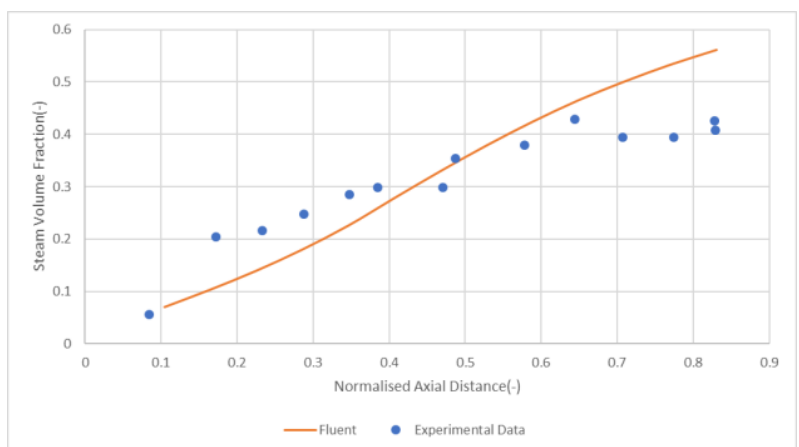

Experiment 3

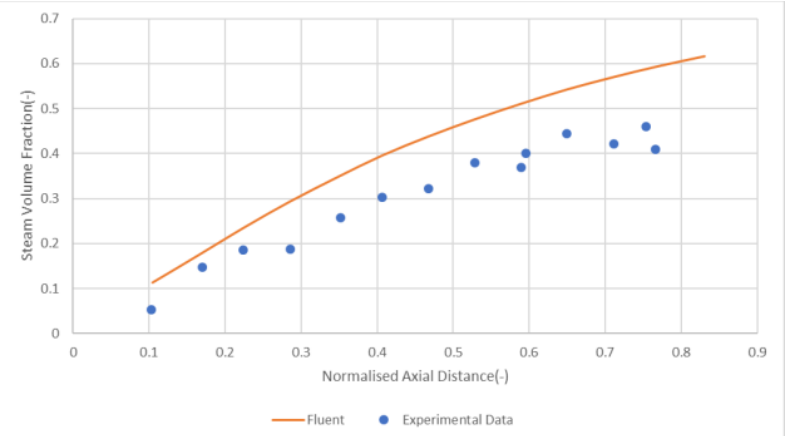

Experiment 7

Fig. 7: Average vapour volume fraction profiles.

\section{Conclusions}

The RPI boiling model has been used to predict nucleating boiling for $2 \mathrm{~d}$, and $3 \mathrm{~d}$ boiling flows. The results demonstrate that the model can correctly predict the average vapour volume fraction in the vertical pipe. In the $3 \mathrm{~d}$ rectangular duct, the average vapour volume fractions are also predicted with reasonable accuracy.

While the RPI model gives good agreement between the predicted and experimental data for nucleate boing flow, some further research and development are required to improve its accuracy and applicability for broader application.

\section{References}

[1] N. Kurul, M. Z. Podowski, "Multidimensional effects in sub-cooled boiling," in Proc. 9th Heat Transfer Conference, Jerusalem, 1990.

[2] G. G. Bartolemei, G. N. Batashova, V. G. Brantov, et.al. In: Teplomassoobmen-IV. Vol.5. Minsk. ITMO AN BSSR Press, 1980, p.38.

[3] G. G. Bartolemei, V. I. Gorburov, "Experimental study of vapour phase condensation in liquid subcooled below saturation temperature," Heat Production, no. 12, pp. 58-62, 1969.

[4] C. C. Pierre, and G. Bankoff, "Vapor volume profiles in developing two-phase flow," Int. J. Heat Mass Transfer, vol. 10, pp. 237-249, Pregamon Press 1967.

[5] Multiphase Flows, Theory Guide, ANSYS FLUENT 19.0 Documentation, 2018. 